Ann. Zootech., I973, 22 (I), 37-54.

\title{
UTILISATION DES LUZERNES ET DES GRAMINÉES DÉSHYDRATÉES COMME UNIQUE FOURRAGE DE LA RATION DES VACHES LAITIÈRES, ET ÉTUDE DE LEUR DIGESTION DANS LE RUMEN
}

\author{
M. JOURNET et A. HODEN \\ avec la collaboration technique de Jeanne Fléchet, B. MarQUis et A. Ollier \\ Station de Recherches sur l'Élevage des Ruminants, \\ Centre de Recherches de Clermont-Ferrand, I. N. R. A., \\ Saint Genès Champanelle, 63110 Beaumont
}

\section{RÉSUMÉ}

Deux essais d'alimentation, l'un avec 3 luzernes, l'autre avec un ray-grass italien, ont permis d'étudier l'influence du mode de conditionnement des fourrages déshydratés comme uniques tourrages sur leur utilisation par les vaches laitières. L'étude du comportement alimentaire et de la digestion a été effectuée sur des vaches fistulées du rumen.

Les luzernes ont été conditionnées sous la forme condensée (broyage du fourrage avec une grille à mailles de $20 \mathrm{~mm}$ et pressage dans une presse à filières), la forme compactée (pressage dans une presse à filières sans broyage préalable) et la forme comprimée (pressage đans une presse à piston sans broyage préalable) ; le ray-grass a été conditionné sous les formes condensée et compactée. La taille moyenne des particules de fourrage a varié selon le mode de conditionnement, de 0,49 à $\mathrm{I}, 25 \mathrm{~mm}$, et le pourcentage en poids de particules supérieures à $\mathrm{I}, 25 \mathrm{~mm}$, de 2 à $4^{8}$ p. I00.

Le niveau d'ingestion avec les luzernes, surtout celles du $\mathrm{I}$ er cycle, a été élevé puisqu'il a atteint $3,3 \mathrm{~kg}$ de matière sèche par $100 \mathrm{~kg}$ de poids vif, et il a été supérieur à celui avec le raygrass $(2,65$ p. Ioo du poids vif). Le mode de conditionnement n'a pas modifié significativement les quantités ingérées (tabl. 2).

Le taux butyreux n'a pas été modifié par le mode de conditionnement. Par rapport aux régimes normaux utilisés au cours des périodes pré-et post-expérimentales, il a été légèrement plus faible dans le cas des luzernes, et légèrement plus élevé dans le cas du ray-grass (tabl. 2).

Les vaches qui ingéraient les luzernes ont été suralimentées en énergie et plus encore en matières azotées; elles ont gagné du poids et la persistance de la production laitière a été bonne. En revanche, avec le ray-grass, la production laitière a diminué assez rapidement et les vaches ont gagné du poids (fig. I).

Les durées de rumination et de mastication (consommation + rumination) et l'activité cellulolytique dans le rumen ont été liées positivement à la taille moyenne des particules de fourrage. Elles ont été accrues par l'ingestion de paille (tabl. 3). La composition du mélange des acides gras volatils du rumen a relativement peu varié avec le mode de conditionnement. Avec les luzernes, elle a été voisine de celle observée avec des rations normales et elle a peu varié en fonction du temps écoulé depuis le début du repas. Avec le ray-grass le rapport $\mathrm{C}_{2} / \mathrm{C}_{3}$ a été faible 3 heures après le repas et la composition du mélange des AGV a varié considérablement 
suivant l'heure de prélèvement ( $\operatorname{tabl}, 4)$. La déviation métabolique observée : accroissement des dépôts corporels aux dépens de la quantité de lait sécrétée, a pu être reliée à ces modifications de Ia composition du mélange des AGV.

Différentes méthodes d'estimation de la valeur énergétique nette des fourrages pour la production laitière ont été comparées. Il semble que le conditionnement diminue moins la valeur énergétique nette que ne le laisserait prévoir la diminution de digestibilité (tabl. 5)

\title{
INTRODUCTION
}

Le conditionnement des fourrages sous forme broyée et agglomérée (forme conđensée) permet d'accroître les quantités ingérées par les vaches laitières et d'augmenter ainsi la part des fourrages dans la ration comme l'ont montré de nombreux essais effectués avec des foins (cf. revue de MOORE, I964 ; JOURNET et JARRIGE, I967 ; JoURNE,T, I970). Mais ce mode de conditionnement réduit le fourrage (surtout s'il est déshydraté) en fines particules, ce qui provoque une diminution de la digestibilité de la matière organique (BLAXTER, et GRAHAM, I956 ; RODRIGUE et ALIEN, I960 ; DEMARQUILLY, I97I), des accidents sanitaires et des baisses du taux butyreux chez les vaches laitières. Pour cette raison, il a été proposé de ne pas broyer le fourrage déshydraté et de le presser directement, soit dans une presse à filières (forme compactée), soit dans une presse à piston (forme comprimée). Mais on connaît mal dans le cas des vaches laitières, 1'influence de ces modes de conditionnement des luzernes et des graminées, et par ailleurs, peu d'essais ont été effectués pour étudier les possibilités d'alimenter les vaches laitières avec des fourrages déshydratés comme seuls fourrages.

Nous avons effectué un premier essai d'alimentation avec des luzernes et un $2^{\text {e }}$ essai avec un ray-grass italien pour connaitre le niveau des apports nutritifs et l'effet de ces rations sur le gain de poids vif, la production et la composition du lait, en particulier le taux butyreux. Simultanément, l'étude de la digestion a été effectuée avec des vaches fistulées du rumen et en bilan digestif.

\section{MATÉRIEL ETT MÉTHODE}

\author{
Essais d'alimentation
}

Schémas expérimentaux.

Luzernes : Les luzernes utilisées ont été récoltées, a) au stade bourgeonnement du Ier cycle, b) au stade floraison du $\mathrm{I}^{\mathrm{er}}$ cycle, c) à 6 semaines d'àge au $3^{\mathrm{e}}$ cycle. Chaque stade a été présenté sous 3 formes différentes : $\left.\mathbf{I}^{\circ}\right)$ condensée après un broyage à la grille de $20 \mathrm{~mm} ; 2^{\circ}$ ) compactée ; $3^{\circ}$ ) comprimée.

Elles proviennent de 2 parcelles; chacune des luzernes récoltées sur la première parcelle a été conditionnée sous les formes condensée et compactée; la déshydratation a eu lieu à haute température dans une déshydrateuse Promill, type S. M. 60o. Chacune des luzernes récoltées sur la $2^{\mathbf{e}}$ parcelle a été conditionnée sous la forme comprimée après avoir été déshydratée à basse température dans une déshydrateuse du type Van der Broek. Le diamètre des agglomérés sous formes condensée et compactée était de $12 \mathrm{~mm}$ et sous forme comprimée de $5^{\circ} \mathrm{mm}$. 
Pour chacune des 3 récoltes nous avons (lonc 3 formes de présentation I) condensée 2) compactée 3) comprimée, soit 9 échantillons de fourrage. La comparaison de ces 3 formes de présentation a été effectuée pour chacune des récoltes en utilisant un dispositif en carré latin $(3 \times 3)$ avec 3 lots de 3 vaches de race F.F.P.N. par récolte, soit 27 vaches au total. Les vaches ont reçu les 3 formes de conditionnement à volonté au cours de 3 périodes successives de 4 semaines. La période expérimentale a été précédée d'une période pré-expérimentale de 3 semaines sur un régime de foin et d'ensilage d'herbe offerts à volon té et de bettcraves offertes en quantitées limitées (20 kg). La période post-expérimentale a été de 6 semaines, les 3 premières sur un régime de foin offert à volonté et les 3 dernières à l'herbe. Les périodes de transition au début et à la fin de la période expérimentale ont duré 2 semaines.

Les vaches utilisées pour étudier les 2 luzernes du r er cycle étaient en moyenne à la fin du $2^{\text {e }}$ mois de lactation au début de l'expérience, et elles ont été appariées; les résultats obtenus pour les 2 premiers stades de luzerne peuvent donc être comparés. Les vaches utilisées pour étudier la luzerne du $3^{\mathrm{e}}$ cycle étaient à un stade de lactation moins avancé (fin du premier mois de lactation au début de l'expérience) et l'expérience a commencé 7 semaines plus tard.

Graminées: Le ray-grass italien a été récolté au stade épiaison du I $^{\text {er }}$ cycle de végétation et déshydraté à haute température avec la même déshydrateuse que les luzernes. La forme condensée, après un broyage à la grille de $20 \mathrm{~mm}$ a été comparée à la forme compactée; le diamètre des agglomérés était respectivement de Io et $12 \mathrm{~mm}$. La comparaison a été effectuée avec 2 lots de 7 vaches en utilisant un dispositif d'inversion des régimes au cours de 2 périodes expérimentales de 3 semaines chacune. L'expérience avait été précédée d'une période pré-expérimentale de 3 semaines sur un régime d'ensilage de maïs et de foin offerts à volonté et de pulpes sèches distribuées en quantité limitée, et a été suivie d'une période post-expérimentale de 3 semaines sur un régime de foin (ad libitum) et de pulpes sèches (quantité limitée). Le passage du régime pré-expérimental au régime expérimental s'est effectué en 2 semaines et celui du régime expérimental au régime post-expérimental en I semaine. Les $\mathrm{I}_{4}$ vaches laitièrcs cle race Frisonne dont 6 étaient en $\mathrm{I}^{\mathrm{re}}$ lactation se trouvaient en moyenne à la $\mathrm{I} 5^{\mathrm{e}}$ scmaine de la lactation au début de la I $^{\text {re }}$ période expérimentale.

\section{Alimentation et mesures.}

Les fourrages déshydratés ont été offerts 2 fois par jour, ad libitum, (ro p. Ioo de refus). Un aliment concentré a été distribué suivant la nécessité et les besoins des vaches; les quantités offertes ont été déterminées chaque semaine selon les apports énergétiques des fourrages déshvdratés, estimés d'après les quantités ingérées mesurées individuellement chaque jour ( 6 jours sur 7 ) et la valeur énergétique des aliments, fournie par les tableaux de la valeur alimentaire des fourrages (DEMARQUiLly et Weiss, I970) (tabl. I). Les vaches laitières de trouvaient sur une litière de paille et pouvaient en consommer.

TABLEAU I

Caractéristiques et composition chimique des fourrages déshydratés selon le stade de vécolte et le mode de conditionnement

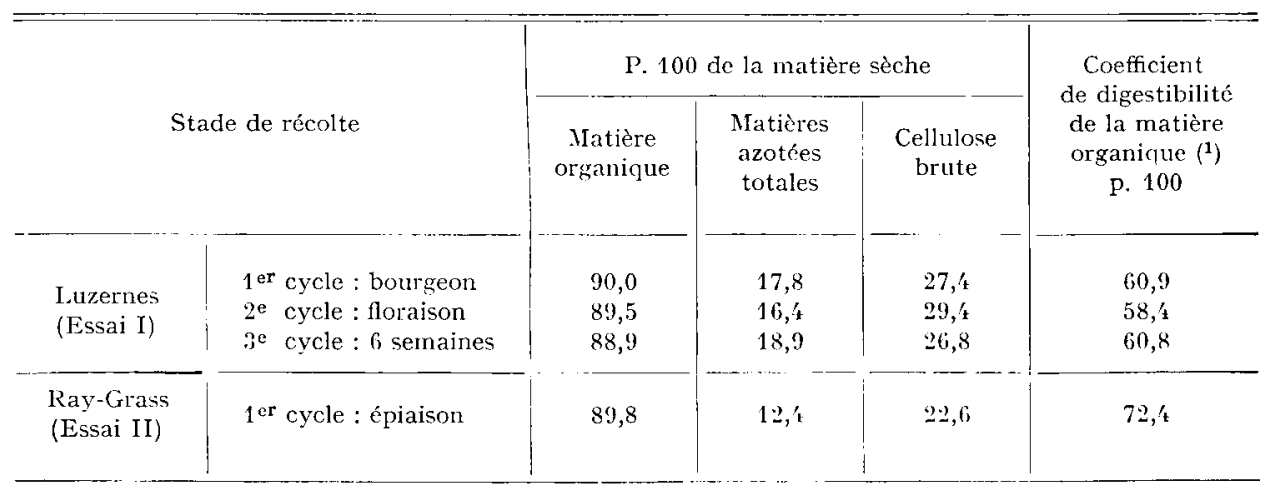

(1) Coefficient de digestibilité mesuré sur les moutons recevant les fourrages déshydratés hachés ad libitum. 
Les quantités de fourrages déshydratés distribuées n'étaient pas réduites quand les apports énergétiques et azotés dépassaient les besoins ce qui fut le cas pour beaucoup d'animaux.

La production de lait et le taux butyreux (méthode Gerber) ont été mesurés individuellement chaque jour et le taux de protéines du lait (noir amido) une fois par semaine. Seuls les résultats obtenus au cours des 2 dernières semaines de chaque période expérimentale ont été pris en considération. Les vaches ont été pesées une fois par semaine à jour et heures fixes.

TABI,EAU I (suite)

\begin{tabular}{|c|c|c|c|c|c|c|}
\hline \multirow{2}{*}{\multicolumn{2}{|c|}{ Mode de conditionnement }} & \multicolumn{2}{|c|}{ p. 100 de matière sèche } & \multirow{2}{*}{$\begin{array}{c}\text { Diamètre } \\
\text { des agglomérés } \\
(\mathrm{mm})\end{array}$} & \multirow{2}{*}{$\begin{array}{l}\text { Taille moyenne } \\
\text { des particules } \\
\text { (mm) }\end{array}$} & \multirow{2}{*}{$\begin{array}{l}\text { P. } 100 \mathrm{de} \\
\text { particules } \\
>1,25 \mathrm{~mm}\end{array}$} \\
\hline & & Matières & Cellulose & & & \\
\hline $\begin{array}{c}\text { Luzernes } \\
\text { (Essai I) }\end{array}$ & $\begin{array}{l}\text { Condensé } \\
\text { Compacté } \\
\text { Comprimé }\left({ }^{1}\right)\end{array}$ & $\begin{array}{l}17,8 \\
17,5\end{array}$ & $\begin{array}{l}26,8 \\
27,9\end{array}$ & $\begin{array}{l}10 \\
12 \\
50\end{array}$ & $\begin{array}{l}0,82 \\
1,12 \\
1,25\end{array}$ & $\begin{array}{l}21,0 \\
34,3 \\
48,5\end{array}$ \\
\hline $\begin{array}{l}\text { Ray-Grass } \\
\text { (Essai II) }\end{array}$ & $\begin{array}{l}\text { Condensé } \\
\text { Compacté }\end{array}$ & $\begin{array}{l}12,4 \\
12,4\end{array}$ & $\begin{array}{l}22,7 \\
22,6\end{array}$ & $\begin{array}{l}10 \\
12\end{array}$ & $\begin{array}{l}0,49 \\
0,77\end{array}$ & $\begin{array}{r}2,0 \\
16,8\end{array}$ \\
\hline
\end{tabular}

(1) Luzerne récoltée sur une parcelle différente.

\section{Étude sur les vaches fistulées}

Deux vaches fistulées du rumen ont été utilisées pour chacune des récoltes de luzerne ct pour celle de ray-grass, dans le but d'étudier l'influence de la forme de présentation sur le comportement alimentaire des animaux, la digestibilité des fourrages et la digestion dans le rumen. Chaque forme de présentation a été offerte ad libitum et en quantité limitée (ajustée aux besoins énergétiques). Seul le ray-grass a été étudié en présence de paille distribuée en plus, ad libitum, ou en absence de paille. La stalle des animaux était constituée de traverses de bois recouvertes de caoutchouc, sans litière de paille.

Les périodes expérimentales ont été de 3 semaines. Les mesures cffectuées au cours de la dernière semaine de chaque période ont été les suivantes : digestibilité par collecte totale des fécès (sur 7 jours) ; enregistrement des temps d'ingestion et de rumination (pendant 4 jours consécutifs), activité cellulolytique du jus de rumen (par la méthode des sachets, Journet et Demargurely, I967) ; teneur et composition en acides gras volatils (A. G. V.) du jus de rumen (o h et 3 heures après le début du repas et pendant 2 jours consécutifs selon la méthode de Rigaud et Journer (1970).

La digestibilité des mêmes fourrages déshydratés sous forme conditionnée ainsi que sous forme hachée a également été mesurée sur des moutons et les résultats ont déjà été rapportés dans une autre publication (DEMarguILly, I97I)

\section{RÉSULTATS}

L'analyse granulométrique effectuée par tamisage à sec après humidification, délitement manuel des agglomérés et séchage à l'étuve, révèle que le fourrage déshydraté a été réduit en particules plus ou moins fines selon le type de presse et selon qu'il a été broyé ou non au préalable.

La taille moyenne des particules a été la plus grande pour la forme comprimée et la plus faible pour la forme condensée; elle a varié de 0,63 à $1,25 \mathrm{~mm}$ et elle 
FOURRAGES DÉSHYDRATÉS POUR VACHES LAITIÈRES

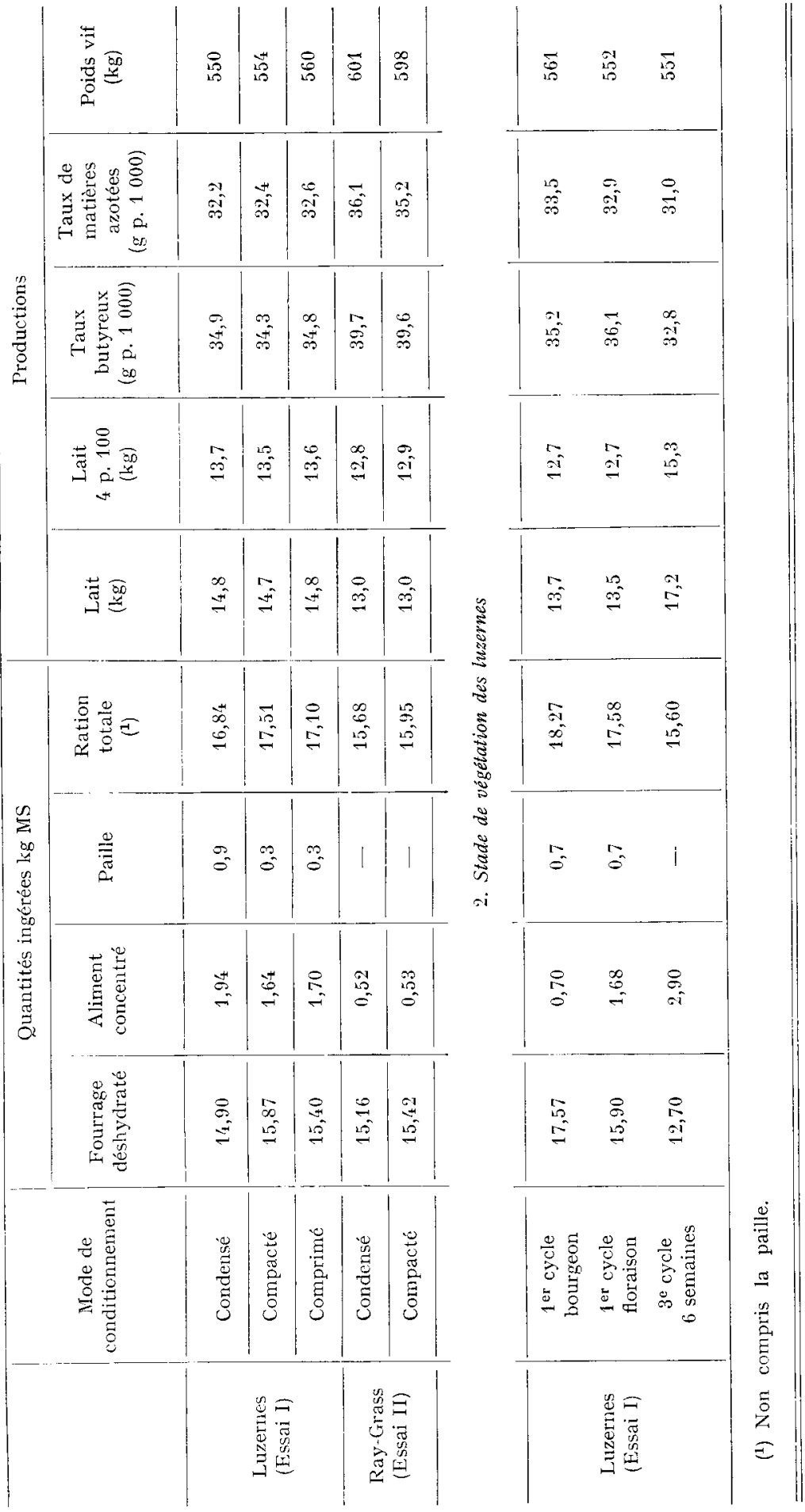


a été iiée étroitement $(r=0,98)$ au pourcentage (en poids) de grosses particules (> I,25mm) qui a varié de 8 à 48 p. Ioo pour les I I échantillons étudiés. Pour un même mode de conditionnement, le ray-grass a été broyé plus finement que les luzernes puisque la taille moyenne des particules a été d'environ la moitié plus faible (tabl. I). Mais d'une façon générale, la taille moyenne des particules de ces fourrages, qui ont été conditionnés par 1'I. T. C. F., est supérieure à celle des fourrages conditionnés par les usines privées.

Les caractéristiques chimiques n'ont pas été différentes selon le mode de conditionnement, condensé ou compacté, de la luzerne ou du ray-grass (tabl. I).

\section{Quantités ingérées, production et composition du lait, gain de poids vif des vaches en essai d'alimentation}

Les quantités ingérées de luzernes du I ${ }^{\text {er }}$ cycle de végétation (moyenne des 3 formes) ont été élevées, 2,9 et 3,I $\mathrm{kg}$ de matière sèche par roo $\mathrm{kg}$ de poids vif (MS par Ioo $\mathrm{kg}$ PV), respectivement pour les stades floraison et bourgeonnement. Elles n'ont été que de $2,3 \mathrm{~kg}$ MS p. Ioo PV avec la luzerne du $3^{\mathrm{e}}$ cycle, probablement en raison des quantités plus élevées d'aliments concentrés et du stade de lactation moins avancé des vaches. Les quantités de luzerne ingérées n'ont pas été influencées significativement par le mode de conditionnement bien qu'elles aient été maximum avec la forme compactée pour les 2 luzernes du $\mathrm{I}^{\mathrm{cr}}$ cycle. Le niveau d'ingestion de la ration totale a atteint près de $3.3 \mathrm{~kg}$ MS p. Ioo PV avec ces dernières (tabl. 2).

Les quantités ingérées de ray-grass déshydraté et, surtout, de la ration totale ont été inférieures à celles obtenues avec les luzernes, mais elles n'ont pas non plus été influencées par le mode de conditionnement condensé ou compacté (tabl. 2).

Avec les luzernes, le niveau des apports azotés a été supérieur aux besoins d'environ $70 \mathrm{p}$. IoO. Le niveau des apports énergétiques a été supérieur aux besoins, principalement dans le cas des vaches faibles productrices, mais il est difficile de savoir de combien en moyenne, en raison de l'incertitude sur la valeur énergétique des fourrages conditionnés (cf. ci-après).

Avec les luzernes, la production de lait et le taux butyreux n'ont pas été influencés par le mode de conditionnement (tab1. 2), mais le taux butyreux a cependant été inférieur de $\mathrm{I}, 6 \mathrm{~g}$ p. I ooo par rapport aux périodes pré- et post-expérimentales sur des régimes de fourrages normaux (fig. I). Cette diminution est hautement significative ; elle est plus importante, mais non significativement, avec la forme compactée $(-2, \mathrm{I}$ g p. I ooo) qu'avec les 2 autres formes condensée et comprimée (respectivement - I,3 et - I,4 g p. I ooo), peut-être en raison du niveau d'ingestion légèrement plus élevé des luzernes compactées. Le taux de matières azotées du lait $(34,4 \mathrm{~g}$ p. I ooo en moyenne) n'a pas été modifié par rapport aux périodes pré- et post-expérimentales, ni influencé par le mode de conditionnement. Le coefficient mensuel de persistance de la production de lait a toujours été élevé et compris entre 9 I et 93 p. Ioo ( fig. I). Le gain de poids vif des animaux entre les périodes pré- et post-expérimentales, pendant lesquelles les animaux recevaient des régimes à base de fourrages normaux a été assez élevé : de 330,330 et I 20 g/jour respectivement pour les 3 luzernes, I er cycle épiaison, $\mathrm{I}^{\text {er }}$ cycle floraison et $3^{\mathrm{e}}$ cycle âgé de 6 semaines.

Avec le ray-grass, la quantité de lait, le taux butyreux et le taux de matières azotées n'ont pas été significativement différents pour les 2 modes de conditionne- 
ment, condensé et compacté. La production de lait de l'ensemble des animaux qui recevaient les fourrages déshydratés a diminué assez rapidement, malgré la suralimentation énergétique, puisque le coefficient mensuel de persistance n'a été que de 89 p. Ioo ; en contrepartie les animaux ont gagné I Io g de poids par jour entre les
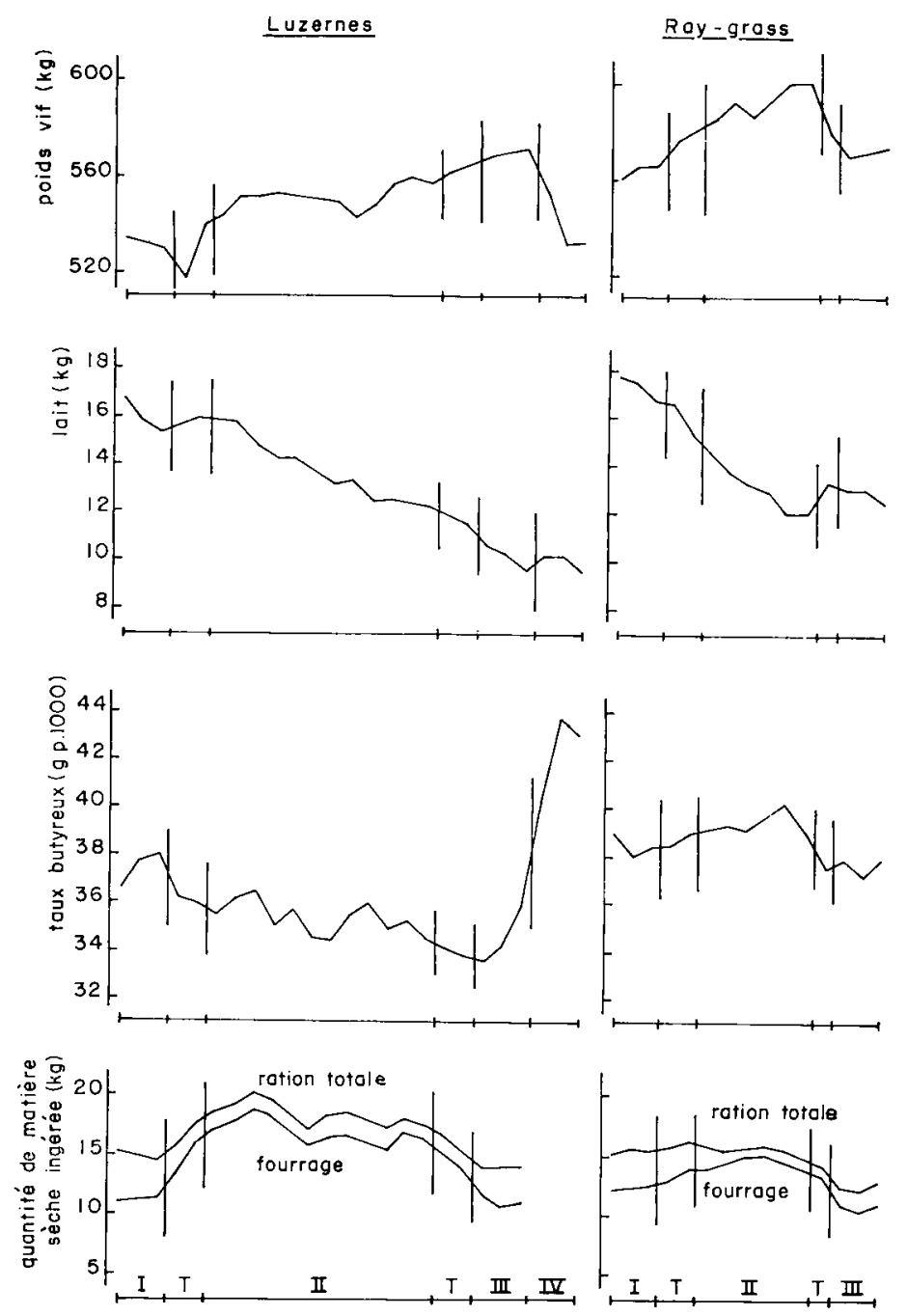

FIG. I. - Utilisation par les vaches laitières de rations de fourrages déshydratês conditionnés (luzernes et ray-grass d'Italie au i er cycle de végétation)

comparativement à des rations normales utilisées au cours des périodes pré-et post-expérimentales

Périodes : II, expérimentale; I et III pré-et post-expérimentales avec des fourrages conservés; IV post expérimentale au pâturage; $T$ de transition

périodes pré- et post-expérimentales (I2 semaines) (fig. I). Par rapport aux périodes pré- et post-expérimentales sur des fourrages normaux, le taux butyreux a été supérieur respectivement de I,O et $\mathrm{I}, 2 \mathrm{~g}$ p. I 000 , et le taux de matières azotées de $\mathrm{I}, \mathrm{O}$ et $0,5 \mathrm{~g}$ p. I 000 . 
M. JOURNET, A. HODEN

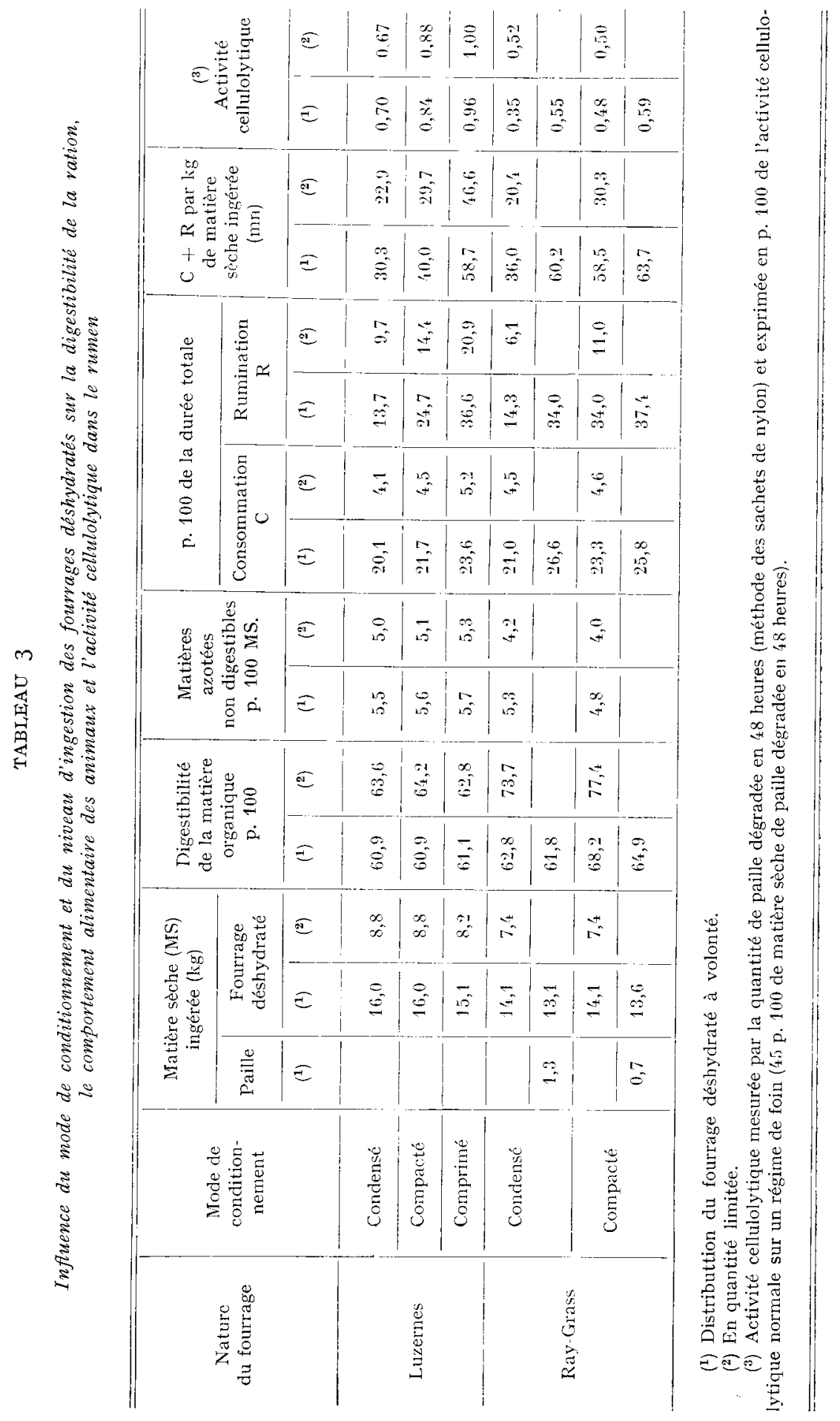




\section{Étude du comportement alimentaire et de la digestion} sur les vaches fistulées

\section{Comportement alimentaire.}

Les durées de rumination par 24 heures et de "mastication 》 unitaires (durée de consommation + rumination par $\mathrm{kg}$ de matière sèche ingérée) des vaches fistulées qui ont reçu les luzernes et le ray-grass déshydratés conditionnés ont été faibles comparativement aux valeurs obtenues avec des foins, et, d'autant plus faibles que le fourrage déshydraté avait été réduit en particules de plus en plus fines en passant de la forme comprimée à la forme condensée (tabl. 3). Ces durées ont été liées très étroitement à la taille moyenne des particules pour les 9 échantillons de luzerne : $r=0,9 \mathrm{I}$ pour la durée de rumination et $r=0,75$ pour la durée de mastication unitaire (minute par $\mathrm{kg}$ de matière sèche ingérée). Elles ont été beaucoup plus élevées avec le raygrass qu'avec les luzernes, à taille de particules égales. Des durées presque normales

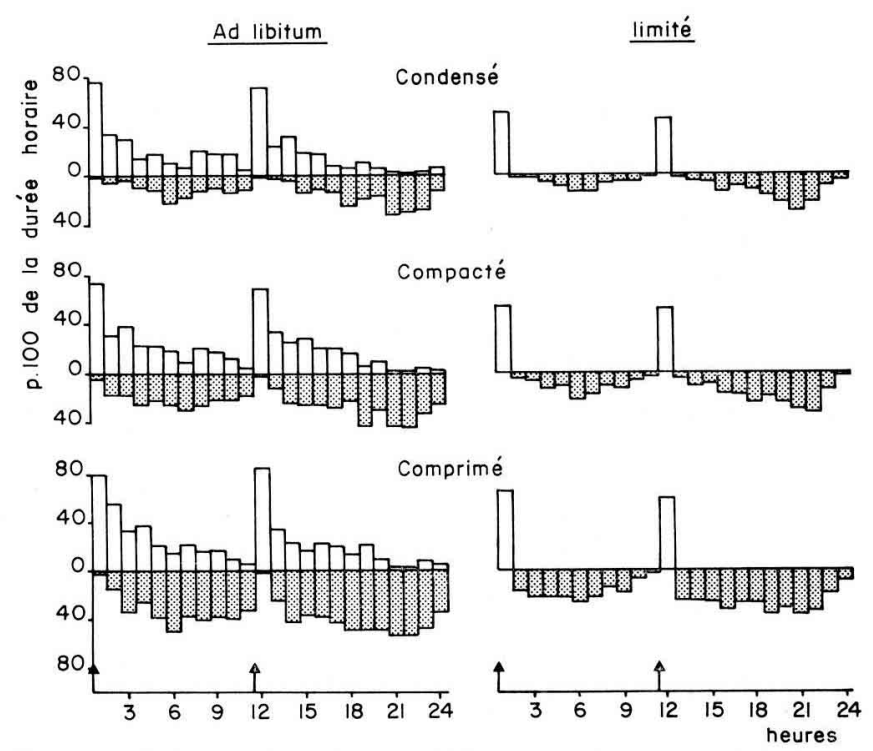

FIG. 2. - Influence du mode de conditionnement des luzernes déshydratées et de la distribution en quantité limitée ou ad libitum sur la répartition nycthémérale des durées de consommation et de rumination $\left.{ }^{(}\right)$

(1) Moyenne des 3 stades avec 3 jours de mesure consécutifs sur 2 vaches par stade et par mode de conditionnement et de distribution

$$
\begin{aligned}
& \uparrow \text { Distribution du fourrage déshydraté } \\
& \square \text { Consommation }
\end{aligned}
$$

de rumination et de mastication unitaires n'ont été obtenues qu'avec la forme comprimée des luzernes et la forme compactée du ray-grass lorsque la taille moyenne des particules était de I,25 et $0,77 \mathrm{~mm}$ respectivement.

Lorsque les vaches ont reçu de la paille à volonté en plus du ray-grass déshydraté, elles en ont ingéré de faibles quantités mais davantage lorsque le ray-grass était condensé $(\mathrm{I}, 4 \mathrm{~kg})$ que lorsqu'il était compacté $(0,7 \mathrm{~kg})$. Le comportement alimentaire 
des animaux qui recevaient l'une ou l'autre forme du ray-grass est alors presque devenu normal et peu différent pour les 2 formes.

La répartition nycthémérale de l'ingestion a été peu modifiée par le mode de conditionnement (fig. 2 et 3 ). Avec ces fourrages agglomérés, la durée d'ingestion a été plus faible qu'avec les fourrages normaux puisqu'elle n'a représenté que 20 et 24 p. Ioo du temps total mais l'ingestion a été bien répartie au cours de la journée et les animaux n'ont pas fait un grand repas après chaque distribution puisque la proportion du temps passé à ingérer était généralement inférieure à $5^{\circ} \mathrm{p}$. roo dès la

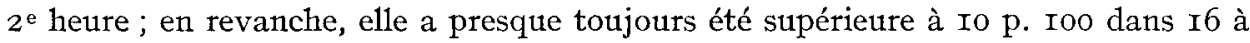
I7 périodes horaires sur 24 .

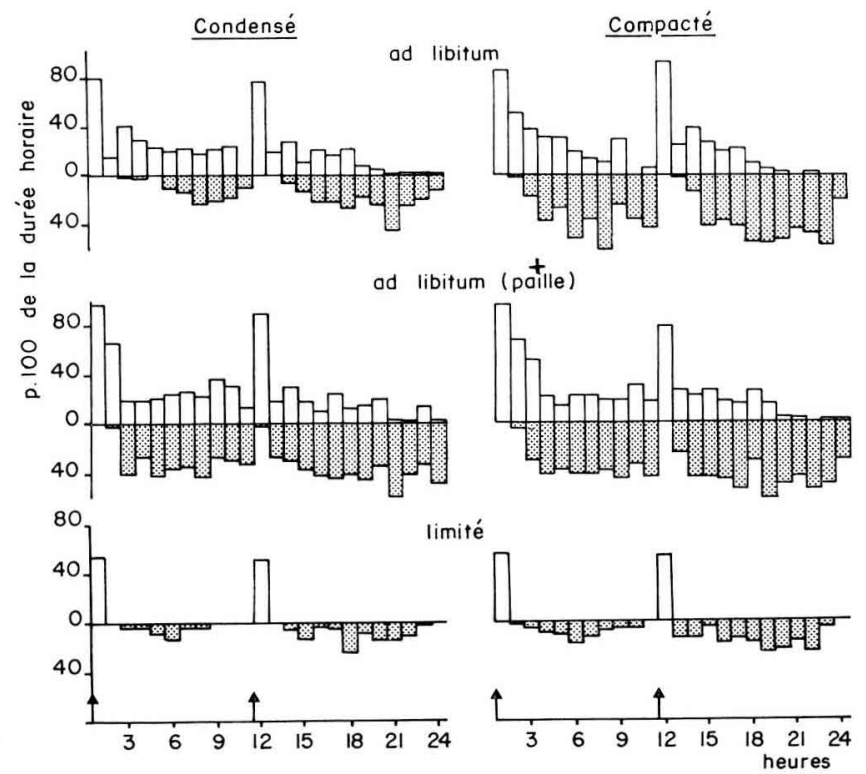

FIG. 3. - Infuence du mode de conditionnement et de la distribution en quantité limitée on ad libitum (avec ou sans paille) d'un ray grass italien déshydraté récolté à l'épiaison du $1^{\text {er }}$ cycle sur la répartition nycthémérale des durées de consommation et de rumination ( $\left.{ }^{1}\right)$. bution.

(1) Moyenne de 3 jours de mesure consécutifs sur 2 vaches par mode de conditionnement et de distri-

$\uparrow$ Distribution đu fourrage déshydraté

Consommation $\quad$ ::: Rumination

La répartition nycthémérale de la rumination a été modifiée par le mode de conditionnement (fig. 2) : avec la forme compactée et surtout comprimée (par rapport à la forme condensée) la rumination a été mieux répartie au cours de la journée ; en particulier la rumination ne présente plus un maximum aussi marqué 6 à ro heures après le début des repas du matin et du soir. On observe la même modification de la répartition nycthémerale de la durée de rumination lorsqu'on ajoute de la paille à la ration de ray-grass condensé (fig. 2). Lorsque le fourrage est distribué en quantités limitées, les vaches ne font qu'un seul repas d'une durée de 20 à 40 minutes après chacune des 2 distributions. La répartition de la rumination est modifiée par le mode de conditionnement, de la même façon que lorsque la distribution a lieu ad libitum. 


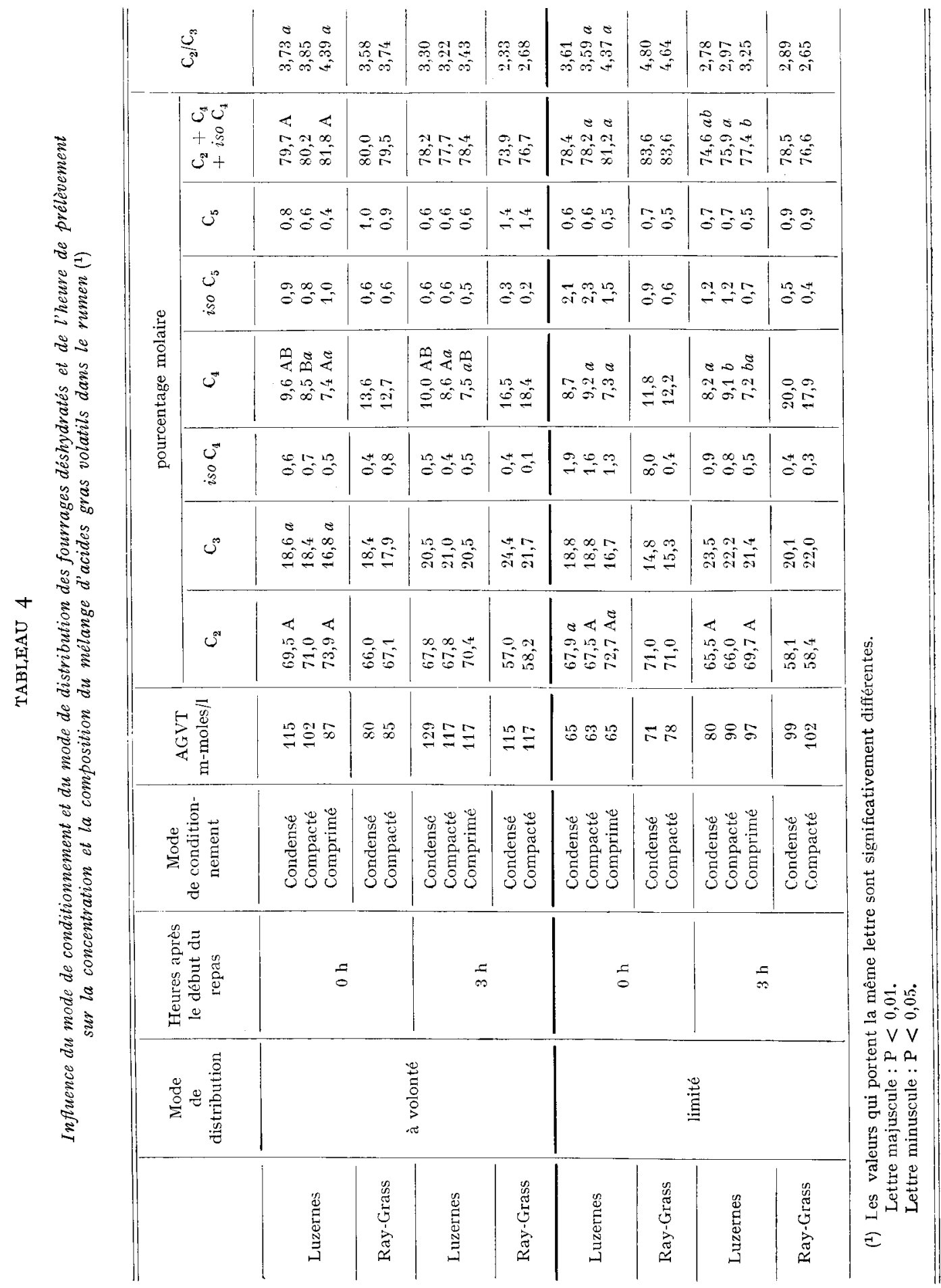


Digestion.

La digestibilité de la matière organique des luzernes distribuées ad libitum n'a pas été influencée par le mode de conditionnement. Celle des luzernes offertes en quantité limitée a été supérieure de 2 à 4 points à celle des luzernes offertes ad libitum. Dans le cas du ray-grass, la digestibilité de la forme condensée a été inférieure à celle de la forme compactée et la différence a été plus grande (de 2,3 points) lorsque la distribution a eu lieu ad libitum (tab1. 3). La digestibilité des matières azotées n'a pas été influencée par le mode de conditionnement; la teneur en matières azotées indigestibles a varié de 4 à 5,7 p. Ioo : elle a été plus faible avec le ray-grass qu'avec les luzernes, et lorsque les quantités de fourrage déshydraté offertes ont été limitées.

La digestion dans le rumen a été modifiée par le mode de conditionnement. En particulier, l'activité cellulolytique est liée étroitement à la taille moyenne des particules du fourrage quelle que soit la nature de celui-ci, au temps de rumination et au temps de mastication, le ray-grass mis à part.

$$
\begin{array}{ll}
\mathrm{Y}=30,89 x_{1}+4,23 & r=0,97 \text { (luzernes }+ \text { ray-grass) } \\
\mathrm{Y}=0,42 x_{2}+26,59 & r=0,95 \text { (luzernes) } \\
\mathrm{Y}=0,28 x_{3}+24,85 & r=0,87 \text { (luzernes) }
\end{array}
$$

$\mathrm{Y}=$ pourcentage de paille dégradée en 48 heures dans le rumen

$x_{1}=$ taille moyenne des particules de fourrage $(\mathrm{mm})$

$x_{2}=$ durée de rumination (p. Ioo en 24 heures)

$x_{3}=$ durée (minutes) de mastication unitaire (durée de consommation + rumination par $\mathrm{kg}$ de matière sèche ingérée).

L'activité cellulolytique est accrue par l'ingestion de paille dans le cas du raygrass condensé, tout en restant inférieure à la normale. Elle est inférieure à celle observée avec les luzernes, quel que soit le mode de conditionnement.

La concentration du mélange des $A G V$ du rumen n'a pas été modifiée par le mode de conditionnement ; elle a varié dans le même sens que le niveau d'ingestion et a été plus élevée 3 heures après le début du repas qu'avant celui-là, sauf pour les luzernes distribuées ad libitum (tabl. 4).

La composition du mélange des $\mathrm{AGV}$ avec les différents fourrages conditionnés utilisés, comparativement à des fourrages normaux, a surtout été modifiée dans le cas du ray-grass. Avec ce dernier le rapport $\mathrm{C}_{2} / \mathrm{C}_{3}, 3$ heures après le début $\mathrm{du}$ repas a été particulièrement faible.

Le mode de conditionnement n'a modifié que faiblement la composition du mélange des $\mathrm{AGV}$, comparativement au comportement alimentaire des animaux et à l'activité cellulolytique dans le rumen. I,es différences observées ont principalement eu lieu entre les formes condensées ou compactées et la forme comprimée des luzernes. Elles ont principalement porté sur les proportions de $\mathrm{C}_{2}$ et surtout de $\mathrm{C}_{4}$ qui ont varié en sens inverse, et sur le rapport $\mathrm{C}_{2} / \mathrm{C}_{3}$ et la somme $\mathrm{C}_{2}+\mathrm{C}_{4}+i$ so $\mathrm{C}_{4}$, qui ont été diminuées par le mode de conditionnement, condensé ou compacté, qui broyait le plus le fourrage (tabl. 4).

En fonction de l'heure de prélèvement, les variations de composition du mélange des $\mathrm{AGV}$ ont été considérables dans le cas du ray-grass et faibles dans le cas des luzernes, principalement lorsque la distribution avait lieu ad libitum (tabl. 4). 


\section{Estimation de la valeur énergétique nette des fourrages déshydratés pour la production laitière}

La valeur énergétique nette des 4 fourrages utilisés a été calculée :

$I^{\circ}$ à partir de la digestibilité de la matière organique, mesurée sur les vaches ou sur les moutons ; les valeurs obtenues sur le fourrage vert et sur le fourrage déshydraté haché ou conditionné ont été comparées. La valeur énergétique du fourrage vert est celle fournie par les tableaux de la valeur alimentaire des fourrages de DEMARQUILLY et WEISS (I970) ; celle des fourrages déshydratés a été calculée par la formule de LEHMANN (I94I) révisée par BREIREM (I954), ou celle de DiJKSTRA avec le facteur correctif de la teneur en cellulose brute $k=0,29$.

$2^{\circ}$ à partir des performances de production des vaches, en utilisant comme valeurs énergétiques nettes $0,38 \mathrm{UF}$ par $\mathrm{kg}$ de lait $4 \mathrm{p}$. Ioo et $4 \mathrm{UF}$ par $\mathrm{kg}$ de gain de poids vif, les vaches ayant gagné du poids quel que soit le fourrage étudié.

\section{TABLEAU 5}

Influence du conditionnement des fourrages déshydratés sur lour valour énorgátique notte (UF/kg $\mathrm{MS}$ ) appréciée selon différentes móthodes

\begin{tabular}{|c|c|c|c|c|c|}
\hline & & \multicolumn{3}{|c|}{ Luzernes } & \multirow{2}{*}{$\begin{array}{l}\text { Ray-Grass } \\
1 \text { cr cycle } \\
\text { épiaison }\end{array}$} \\
\hline & & $\begin{array}{l}1^{\text {cr }} \text { cycle } \\
\text { bourgeon }\end{array}$ & $\begin{array}{l}1 \text { er cycle } \\
\text { floraison }\end{array}$ & $\begin{array}{l}3^{\mathrm{e}} \text { cycle } \\
6 \text { semaines }\end{array}$ & \\
\hline \multicolumn{2}{|c|}{ D'après la valeur du fourrage vert initial (1) } & $0,6 x^{\prime}$ & 0,51 & 0,62 & 0,75 \\
\hline \multirow{2}{*}{$\begin{array}{l}\text { D'après la digestibi- } \\
\text { lité des fourrages } \\
\text { déshydratés (2) }\end{array}$} & $\begin{array}{c}\text { Forme hâchée } \\
\text { Moutons }\left({ }^{2}\right) \ldots \ldots \ldots \ldots \ldots\end{array}$ & 0,55 & 0,48 & 0,55 & 0,75 \\
\hline & $\begin{array}{c}\text { Forme conditionnée } \\
\text { Moutons }\left({ }^{2}\right) \ldots \ldots \ldots \ldots \ldots \ldots \\
\text { Vaches }\left({ }^{2}\right) \ldots \ldots \ldots \ldots \ldots \\
\text { Vaches }\left({ }^{3}\right) \ldots \ldots \ldots \ldots \ldots\end{array}$ & $\begin{array}{l}0,52 \\
0,56 \\
0,67\end{array}$ & $\begin{array}{l}0,43 \\
0,48 \\
0,58\end{array}$ & $\begin{array}{l}0,17 \\
0,53 \\
0,61\end{array}$ & $\begin{array}{l}0,59 \\
0,61 \\
0,74\end{array}$ \\
\hline \multicolumn{2}{|c|}{ D'après les essais d'alimentation des vaches $\left(^{\boldsymbol{4}}\right)$} & 0,54 & 0,53 & 0,60 & 0,63 \\
\hline \multirow{2}{*}{$\begin{array}{l}\text { Production et gain } \\
\text { de poids vif des } \\
\text { vaches laitieres }\end{array}$} & Gain de poids vif $(g / j) \ldots$ & 330 & 330 & 130 & 113 \\
\hline & $\begin{array}{l}\text { Coefficient mensuel de per- } \\
\text { sistance de la production } \\
\text { laitic̀re } \ldots \ldots \ldots \ldots \ldots \ldots\end{array}$ & 92,0 & 92,0 & 89,0 & 84,0 \\
\hline
\end{tabular}

(1) Valeurs tirées des tableaux de la valeur alimentaire des fourrages (Demarouilly et Weiss, 1970).

(2) Formule de Breirem : UF/kg MS $=\frac{2,36 \text { MOI) }-1,2 \text { MOND }}{1650}$.

(3) Formule de Dijkstra : UF/kg MS $=$ (MOD $-0,06 \mathrm{MAD}-k \mathrm{CB}) \times 1,43 \quad k=0,29$.

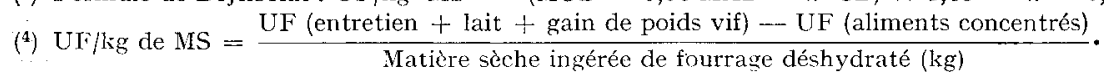


La valeur énergétique nette du fourrage déshydraté haché mesurée sur les moutons a été inférieure à celle du fourrage vert à l'exception du ray-grass ; celle đu fourrage déshydraté conditionné a encore été inférieure à celle đu fourrage haché, surtout dans le cas du ray-grass. On obtient avec les vaches fistulées des valeurs supériettres à celles observées avec les moutons. La formule de Dijkstra en utilisant le facteur $k=0,29$, donne des valeurs très supérieures à celle de Breirem.

En comparant les valeurs obtenues à partir de la mesure de la digestibilité des fourrages déshydratés conditionnés (formule de Dijkstra) à celles obtenues sur les vaches laitières à partir des essais d'alimentation, on observe que ces dernières sont généralement supérieures et qu'elles se rapprochent davantage des valeurs obtenues à partir de la digestibilité du fourrage haché ou des valeurs du fourrage vert (tab1. 5).

\section{DISCUSSION}

Le conditionnement des luzernes n'a pas entraîné de baisse de la valeur énergétique nette pour la production laitière, ce qui doit être dî au broyage relativement grossier du fourrage, en raison d'une technologie de la fabrication parfaitement maîtrisée.

DEMARQUilly (I97I), avait observé sur ces mêmes échantillons de fourrage une diminution de la digestibilité de la matière organique due au conditionnement, de 2,5 points avec les luzernes et de 6,5 points avec le ray-grass ; or, la valeur énergétique pour la production laitière, soit ne diminue pas (avec les luzernes), soit diminue plus faiblement (avec le ray-grass) que ne le laisserait prévoir la baisse de digestibilité.

Par conséquent, on sous-estime la valeur énergétique des fourrages conditionnés pour la production de lait lorsque cette valeur est calculée à partir de la mesture de la digestibilité en utilisant la formule de BREIREM (I954), proposée par R. JARRIGE (I970), pour l'établissement des tableaux de la valeur alimentaire des fourrages. Ces résultats sont en accord avec ceux obtenus par BÉRANGER (I970), sur des taurillons. et ceux de Dijkstra (I970) sur des génisses. C'est la raison pour laquelle ce dernier à proposé d'utiliser pour mesurer la valeur énergétique de ces fourrages (en unités amidon), la formule de Kellner avec le facteur correctif de la teneur en cellulose brute $k=0,29$ au lieu du coefficient $k=0,53$ normalement utilisé et qui donne pour les fourrages, les foins principalement, des valeurs semblables à la formule de Breirem.

Les résultats de nos essais montrent cependant que la correction ainsi apportée pour estimer plus exactement la valeur de production des fourrages déshydratés conditionnés est trop importante dans 3 cas sur 4 et donc que le coefficient $k=0,29$ est trop faible.

Il est probable que les pertes accrues d'énergie dans les fécès, dues au conditionnement des fourrages déshydratés ont été compensées par des pertes plus faibles d'énergie sous forme de chaleur de fermentation et de méthane comme l'ont montré Blaxter et Graham, (I956). L'efficacité d'utilisation métabolique du mélange des $\mathrm{AGV}$ produits dans le rumen n'a pas dû être modifiée dans le cas des luzernes car la composition du mélange des $A G V$ est restée normale. Il n'en est pas de 
même dans le cas du ray-grass dont l'énergie ingérée a par ailleurs surtout servi à accroitre les dépôts corporels aux dépens des quantités de lait et de matières grasses sécrétées par la mamelle; cette déviation métabolique a correspondu à une valeur faible du rapport $C_{2} / C_{3}$ dans le rumen, comme nous l'avons observé avec des rations à forte proportion de céréales (RÉmoND et JoURNET, I97I). ØRskov et al., (I969) ont d'ailleurs montré que l'acide propionique et l'acide acétique ont un effet inverse sur l'activité de synthèse de la mamelle et sur l'adipogenèse.

Il est également possible que l'efficacité d'utilisation de ces luzernes déshydratées conditionnées ait été améliorée du fait qu'une part plus importante de la digestion de ces fourrages broyés a eu lieu dans l'intestin et que les produits terminaux formés ont été mieux utilisés; ce pourrait être le cas des matières azotées dont le niveau d'apport (avec les luzernes, en particulier) a été élevé.

Les modifications du comportement alimentaire des vaches qui ont reçu les fourrages déshydratés conditionnés ad libitum sont semblables à celles rapportés antérieurement sur les ovins et les bovins qui recevaient des foins broyés et agglométés (Beranger et Jarrige, I962; Campling et Freer, I966; RuCkebush et MarqueT, I963 ; Journet et Demarquili,y, I967) : diminution de la durée d'ingestion et de rumination par $\mathrm{kg}$ de matière sèche ingérée et parfois accroissement du nombre de repas au cours de la journée. Ces modifications ont pu être reliées très étroitement à la taille moyenne des particules du fourrage avec cependant des différences entre luzernes et graminées. La taille moyenne des particules du fourrage a également été liée à certains phénomènes de la digestion dans le rumen, en particulier à l'activité cellulolytique, mais paradoxalement, elle n'a que très peu modifié la composition du mélange des $\mathrm{AGV}$ du rumen, tout au moins pour une même espèce fourragère. Par rapport à des fourrages sous forme normale, la composition du mélange d'AGV est restée presque inchangée dans le cas des luzernes, contrairement aux observations de Wright et al. (I963), JoRgensen et Schulitz (1963) et ERIKSSON et al. (I968), mais conformément aux résultats que nous avons obtenus antérieurement avec des foins de luzerne broyés et agglomérés (JOURNET et DEMARQUIL,, Y I I67). Cette particularité doit être attribuée au broyage grossier du fourrage et au mode de pressage qui a donné des agglomérés friables, de sorte que la taille moyenne des particules du fourrage a été assez élevée ( 2 I à 48,5 p. roo de particules supérieures à $\mathbf{I}, 5 \mathrm{~mm}$ ). Elle a été supérieure à celle rapportée par Errrksson et al. (I968), qui ont observé une diminution de la proportion d'acide acétique de Io points, par rapport à une ration normale, lorsque la proportion de particules supérieures à I mm de la luzerne déshydratée conditionnée n'était que de II p. Ioo.

L'accroissement du pourcentage d'acide propionique aux dépens de l'acide acétique que nous avons obtenu avec le ray-grass déshydraté, comparativement aux luzernes, correspond bien à une plus faible taille des particules et à une activité cellulolytique plus réduite.

La légère diminution du taux butyreux due au conditionnement des luzernes déshydratées a été plus faible que celle observée par JoRgEnsen et Schur,Tz, (I963) et ERIKSSON et al. (I968), mais elle correspond bien à des modifications peu importantes de la proportion des AGV dans le rumen. Les différences entre les différents modes de conditionnement des luzernes ont été également faibles.

Avec le ray-grass déshydraté, comparativement à des fourrages normaux, le taux butyreux a été paradoxalement élevé malgré une réduction très importante 
de la taille des particules du fourrage, de la durée de rumination et de l'activité cellulolytique du jus de rumen et de la proportion d'acide acétique au profit de l'acide propionique dans le rumen. On doit probablement attribuer ce taux butyreux élevé à la forte proportion d'acide butyrique et à la diminution assez rapide de la production de lait.

Quel que soit le mode de conditionnement, avec une très bonne technique de fabrication des fourrages déshydratés comme celle qui a été utilisée, il semble donc possible d'alimenter des vaches laitières avec des luzernes déshydratées offertes ad libitum. L'apport énergétique des luzernes permet d'assurer une production de I $2 \mathrm{~kg}$ de lait à $4 \mathrm{p}$. Ioo avec la luzerne récoltée à la floraison, et de I $6 \mathrm{~kg}$ avec celle récoltée au stade bourgeonnement $\mathrm{du} \mathrm{I}^{\mathrm{er}}$ cycle. L'apport de matières azotées a été de 70 p. Ioo supérieur aux besoins. De telles rations permettraient done d'alimenter des vaches laitières à production élevée avec de faibles quantités de céréales. Mais un système simplifié de distribution en libre service provoquerait une suralimentatation d'un nombre important de vaches et ne serait concevable qu'avec des troupeaux à haut potentiel : les réserves corporelles accumulées en fin de lactation seraient utilisées au début de la lactation suivante, ce qui permettrait de réduire 1'apport de concentré à cette période. On sait en effet qu'une réduction de la quantité d'aliment concentré offerte est compensée par une augmentation presque équivalente de 1a quantité de fourrage aggloméré ingérée (JOURNET, I968) et que les vaches fortes productrices peuvent utiliser efficacement leurs réserves corporelles au début de la lactation (Mor: et al., I970).

En revanche, le conditionnement des graminées sous quelque forme que ce soit : condensée ou compactée, semble beaucoup moins bien convenir : il provoque non seulement une diminution de la valeur énergétique du fourrage mais aussi une déviation de l'utilisation de l'énergie avec comme conséquence une diminution anormalement rapide de la production laitière et un engraissement des animaux.

La durée des essais expérimentaux de 7 à I2 semaines est insuffisante pour mesurer les effets éventuels à long terme de ces fourrages déshydratés sous forme conditionnée sur la reproduction et sur la santé des animaux. Mais BROCHART (I97I) a observé qu'un excès de matières azotées, surtout avec des luzernes déshydratées, pouvait diminuer la fécondité des vaches laitières.

Reçu pour publication en juin 1972.

\section{SUMMARY}

UTILIZATION OF DRIED LUCERNE, AND GRASS AS SOLE FORAGE IN THE RATION OF DAIRY COWS AND STUDY OF THE DIGESTION IN THE RUMEN

Two feeding trials, one with 3 lucerne crops and the other with I Italian rye-grass, were carried out to study the effect of the mode of processing dried forages on their utilization by dairy cows which received these crops as sole forage. Study of feeding behaviour and digestion was performed on cows fitted with rumen fistulae.

The lucerne crops were processed so as to prepare diets of three different physical forms: pellet (forage ground through a $20 \mathrm{~mm}$ screen and pressed in a pellet mill), cob (forage pressed in a pellet mill without previous grinding) and wafers (forage pressed in a piston press without 
previous grinding). The rye-grass was processed in the pelleted and cob forms. The mean particle size of the forage varied form 0.49 to $1.25 \mathrm{~mm}$ according to the mode of processing ard the weight percentage of particles excecding $1.25 \mathrm{~mm}$, from 2 to $48 \mathrm{p}$. 1 oo.

The total feed intake level with the lucerne crops, especially those of the Ist cycle, was high and reached $3.3 \mathrm{~kg}$ dry matter per $100 \mathrm{~kg}$ live weight, exceding that with the rye-grass $(2.56 \mathrm{p}$. I co of the live weight). The mode of processing did not significantly change the feed intake level (tabl. 2).

The fat content of milk was not modified by the mode of processing the forages. Compared with the normal diets used during the pre and post experimental periods, it was slightly lower for lucerne and a little higher for rye-grass (tabl, 2).

The lucerne diets led to energy and, even more, protcin overfeeding of the cows, which grew in weight and showed a good persistence of the milk production. On the other hand, the rye-grass caused a rather rapid fall of the milk production and the cows gained in weight (fig. I).

The daily time spent cating and chewing (cating and ruminating) and the cellulolytic activity in the rumen were highly correlated to the mean particle size of the forage. They increased when the animals were fed straw (tabl. 3). The mode of processing did not affect much the composition of the volatile fatty acid mixture in the rumen. In the case of lucerne, this composition was similar to that observed with normal diets and it did not differ very much according to the elapsed time since the beginning of the meal. In the case of ryc-grass, the $C_{2} / C_{3}$ ratio was low 3 hours after the meal and the composition of the VFA mixture varied considerably according to the hour of sampling (tabl. 4). The metabolic deviation observed .e. increase of body reserves at the expense of the amount of milk secreted, can be related to these changes of the composition of the VFA mixture.

Different methods for estimating the net energy value of the forages for milk production have been compared. It seems that processing the forage decreases less the net energy value than what could be predicted from the decrease of the digestibility (tabl. 5).

\section{RÉFÉRENCES BIBLTOGRAPHIQUES}

Bérangter C., Jarrigt: R., I962. Utilisation des aliments broyés et agglomérés par les bovins. I. Comparaison du foin de luzerne normal et du foin de luzerne brové dans l'alimentation du bouf à l'engrais. Ann. Zootech., 11, 273-294.

Béranger C., I97o. Utilisation des fourrages déshydratés pour la production de viande bovine. Commission de Production Bovine 21 réunion annuclle de la Fédération européenne de Zootechnie. Gödöllö, Budapest. 24-28 août I970.

Blaxter K. L., Graham N. M. C., I 956. The effect of grinding and cubing process on the utilization of the energy of dried grass. J.Agric. Sci., 47, 207.

Brochart M., I97i. Pathologie de la stérilité liée à la Nutrition. Schteizer Archiv für Tierheilkunde, $113,3-8$.

Campling R. C., Freer M., I966. lactors affecting the voluntary intake of food by cows. Fxperiments with ground, pelleted roughages. Brit. J. Nutr., 20, 229-243.

Demarguilly C., Weiss Ph., r97o. Tableaux de la valcur alimentaire des fourrages S. E. I., Étude $n^{\circ} 42$.

Demarglilly C., i97I. Influence de la déshydratation et du conditionnement sur la valeur alimentaire des fourrages pour le ruminant. Journées d'études internationales de la III'e section de la Commission internationale du Génie Rural. Paris 20-23 juillet, p. 74-89.

Dijkstra N. D., I970. Estimation of nutritive value of artificially dried grass for ruminants. Commission de production bovine, $21^{\mathrm{e}}$ réunion annuelle de la Fédération européenne de Zootechnie. Gödöllö, Budapest, 24-28 août.

Eriksson S., Jonsson G., Persson S. J., Wallin O., Ig68. The influence of pelleted and wafered roughage on the rumen digestion, the milk fat content and the health of cows. Acta. Agr. Scand., 18, I68-176.

Jarrige R., I970. Tableaux de la valeur alimentaire des fourrages de C. Demarquilly et Ph. Weiss. S. E. I. Étude $n^{\circ} 42$.

Jorgensen N. H., Schultz L. H., i963. Ration effects on rumen acids, ketogenesis and milk composition. I. Unrestricted roughage feeding. J. Dairy Sci., 46, 437-443.

Journet M, Demarovilly C., r967. Valeur alimentaire des foins condensés. II. Influence du broyage et de la mise en agglomérés sur la digestion du foin de luzerne dans le rumen. Ann. Zootech., 16, 307-321.

Journet M., JARRIGE R., I967. Utilisation des aliments broyés et agglomérés par les bovins. II. Utilisation comparé par la vache laitière du foin de luzerne condensé et du foin de luzerne normal associés à de l'ensilage et des betteraves. Ann. Zootech., 16, 27I-289. 
JoURnet M., I968. Rations agglomérées pour les vaches laitières. Association franfaise de Zootechnie - Productions de ruminants. Rapport $\mathrm{n}^{\circ} 37$ p. I-9.

Journet M., I970. Utilisation des aliments condensés par les vaches laitières. Ann. Zootech., 19, 85-87.

Moe P. W., Tyrell H. F., Flatt W. P., I970. Partial efficiency of energy use for maintenance, lactation, body gain and gestation in the dairy cow. 5th Symposium on energy metabolism of farm animals, Vitsnaw.

Moore L. A., r964. Symposium on forage utilization : nutritive value of forage as affected by physical form. I. General principles involved with ruminants and effect of feeding pelleted or wafered forage to dairy cattle. J. Anim. Sci., 23, 230-238.

Orskov E. R., Flatt W. P., Moe D. W., Munson A. W., x969. The influence of ruminal infusion of volatile fatty acids on milk yield and composition and on energy utilization by lactating cows. Brit. J. Nut., 23, 443-453.

RÉmond B., JouRnet M., I971. Alimentation des vaches laitières avec des rations à forte proportion d'aliments concentrés. I. Quantités ingérées et production laitière. Ann. Zootech., 20, I69-I84.

Rigaud J., Journet M., I97o. Méthode de dosage des acides gras volatils dans le liquide du rumen. Ann. Biol. anim. Bioch. Biophys., 10, I5I-I 57.

Rodrigue C. B., Allen N. N., 1960. The effect of fine grinding of hay on ration digestibility, rate of passage and fat content of milk. Can. J. Anim. Sci., 50, 23-29.

Ruckebusch Y., Marquet J.-P., i963. Recherche sur le comportement alimentaire chez les ruminants. I. Influence de la structure physique des aliments. Rev. Méd. vét., 114, 833-335.

Schmekel J., I970. The effect of processing different artificially dried forages on apparent digestibility and digestion in the rumen. Commission de production bovine. $21^{\mathrm{e}}$ réunion annuelle de la Fédération européenne de Zootechnie. Gödöllö, Budapest 24-28 août I97o.

Wright P. L., Pope A. L., Philips Ph., I963. Effect of physical form of ration upon digestion and volatile fatty acid production in vivo and in vitro. J. Anim. Sci., 22, 586-59I. 